\title{
DIEZ AÑOS DE INVESTIGACIÓN EN DIDÁCTICA DE LAS CIENCIAS: REALIZACIONES Y PERSPECTIVAS ${ }^{*}$
}

\author{
GIL PÉREZ, D. \\ Departament de Didàctica de les Ciències Experimentals. Universitat de València.
}

\section{SUMMARY}

This paper summarizes the research in science education undertaken by the international community during the past decade, paying a special attention to the Spanish production. We intend to show that science education has improved considerably during this period, becoming a coherent body of knowledge, and we present what could be, in our opinion, the main trends for the next future.

\section{UNA RECAPITULACIÓN NECESARIA}

Éste es un trabajo "de encargo", promovido -al igual que el de Marco Antonio Moreira «Diez años de Enseñanza de las Ciencias" (Moreira 1994) o el de Carlos Furió «Tendencias actuales en la formación del profesorado de ciencias» (Furió 1994)- por el Consejo de Redacción de esta revista con ocasión de sus primeros 10 años de publicación. No se trata, sin embargo, de unos trabajos de circunstancias «al servicio de la celebración» sino que, muy al contrario, se tomó como pretex to la efemérides para satisfacer una necesidad de recapitulación que muchos sentíamos y que la revista consideró necesario potenciar. Las recapitulaciones constituyen, en efecto, una exigencia de todo trabajo con aspiración cientffica, aunque no tiene por qué coincidir con ninguna fecha señalada: se trata de distanciarse periódicamente de las investigaciones concretas en las que cada uno de nosotros estamos implicados para intentar adquirir una visión más amplia y nuevas perspectivas para seguir avanzando.

Sin đuda, 10 años son tiempo más que suficiente para intentar construir una visión global de dónde estamos y cuáles son las perspectivas. Agradecemos, pues, al Consejo de Redacción de Enseñanza de las Ciencias la ocasión que nos ha brindado de colaborar en esta tarea. Pero se trata de una actividad demasiado importante para que quede en manos de unas pocas personas que, inevitablemente proporcionarán una visión sesgada. Sería muy útil, por el contrario, confrontar distintas visiones globalizadoras, que podrán coincidir y apoyarse mutuamente en bastantes aspectos, pero que también diferirán en otros, proporcionando entre todos una visión más rica. Seria muy importante, pues, que otros compañeros y compañeras «reaccionaran» a estos intentos de globalización, manifestando sus điscrepancias ( $y$, por qué no, sus acuerdos) y ofrecieran otros puntos de vista. Podriamos contribuir así, desde las páginas de Enseñanza de las Ciencias, a la revisión necesaria de un período que coincide prácticamente con el despegue de la investigación en didáctica de las ciencias en muchos pafses de Europa y América Latina.

En efecto, a comienzos de la década de los 80 , un breve análisis de la situación de la investigación sobre didáctica de las ciencias en España nos ilevaba a reconocer un vacío prácticamente total (Gil 1982): no existían revistas españolas que pudieran servir de comunicación e impulso y las publicaciones internacionales eran desconocidas; las facultades de Ciencias rechazaban, o simplemente ignoraban, los problemas educativos como temas de investigación y elaboración de tesis; los currículos de formación cel profesorado, no sólo no incluían ninguna preparación a la investigación educativa sino que no hacían la menor referencia a la misma; no se conocía, por último, ningún equipo -más allá de algunas inđividualidades aisladas-con dedicación sistemática al desarrollo de dicha investigación.

Al mismo tiempo, sin embargo, se detectaban otros signos que permitían concebir la posibilidad de un rápido desamollo de la investigación en la didáctica de las 
ciencias. En efecto, a lo largo de los años 70, se habían constituido, particularmente en la enseñanza secundaria, numerosos grupos de trabajo preocupados por el creciente fracaso escolar -asociado al acceso de amplias capas de población a los estudios secundarios- y deseosos de introducir e intercambiar propuestas innovadoras más efectivas. Dicho trabajo se realizó durante más de una década, desconociendo los esfuerzos precedentes de la comunidad científica internacional, sus logros y sus fracasos, lo que limitó, sin duda, su efectividad. Pero el dinamismo y la continuidad de muchos de dichos grupos - cuyo trabajo de renovación se enmarcaba en la lucha por la transformación democrática de la sociedad española-condujo a una profundización en el tratamiento de los problemas que sólo podía encontrar respuesta en la asociación de la innovación con la investigación.

De hecho, como trataremos de mostrar, durante estos 10 años se ha producido, en nuestro país y en la comunidad internacional, un desarrollo espectacular de la investigación didáctica que obliga a una síntesis recapituladora, si queremos evitar que algunos árboles nos escondan el bosque y sacar todo el provecho posibie del enorme esfuerzo realizado durante este período.

\section{UN DESARROLLO CUANTITATIVO ES- PECTACULAR}

Comenzaremos refiriéndonos al notable incremento cuantitativo experimentado por la investigación en didúctica de las ciencias durante estos años. Que esto no es una afirmación gratuita nos lo pueden mostrar toda una seric de índices, como la evolución del número de trabajos publicados o incluso de los órganos de expresión existentes, la datación de los artículos considerados hoy clave, etc. En todos estos casos, el gráfico de la evolución en el tiempo del índice elegido se ajusta a un patrón exponencial. Y ello tanto para la comunidad internacional en general como para países con un desarrollo tardío como el nuestro.

Así ocurre, por ejemplo -como ha mostrado Reinders Duit (1993)-, con el número de trabajos publicados, desde los años 60 hasta nuestros días, sobre el tema específico de las concepciones de los alumnos. Por nuestra parte (Carrascosa, Gil y Martínez-Terrades 1993), hemos analizado más de 20.000 referencias de artículos publicados, sin seleccionar ningún tema, obteniendo también una clara distribución exponencial.

Si analizamos, por otra parte, la evoluctón de los órganos de expresión nos encontramos con que Science Education aparece en 1916, Journal of Research in Science Teaching ya en 1963 y Studies in Science Education en 1972, mientras que en la década de los 80 aparecen numerosas revistas como European Journal of Science Education, Enseñanza de las Ciencias, The Australian Journal of Science Education, ASTER, Science and Technological Education, Revista de Enseñanza de la Física, O Ensino de Física, Investigación en la Escuela, etc., llegando hasta la aparición de revistas especializadas en aspectos concretos como Science \& Education (aparecida en 1991), destinada al estudio del papel de la historia y filosofía de las ciencias en la enseñanza de las ciencias o Aliage, publicada desde 1989 y centrada en las interacciones cultura-ciencia-técnica.

Este proceso de creación de nuevos vehículos de expresión e intercambio continúa pujante. Podemos así mencionar, por ejemplo, la reciente aparición en 1993 de una nueva revista de expresión francesa Didaskalia y, en España, el anunciado larizamiento para 1994 de la revista Alambique. Didactica de las Ciencias Experimentales.

Al mismo tiempo, revistas educativas de carácter más general, como Infancia y Aprendizaje, Cuadernos de Pedagogia, Bordón, Revista de Educación - por citar tan sólo las espariolas- o centradas en los contenidos cientificos, como Bulletin de l'Union des Physiciens, Jour. nal of Chemical Education, Revista Española de Fisica, etc. publican con frecuencia creciente trabajos de innovación e investigación en didáctica de las ciencias.

Se obtiene unia visión igualmente dinámica al analizar las referencias manejadas: los artículos clave, aquéllos que aparecen reiteradamente citados, corresponden en general a aulores cuya actividad investigadora se ha desarrollado básicamente en estos últimos años. De hecho, autores como Aikenhead, Astolfi, Driver, Duit, Duschl, Gilbert, Hewson, Hodson, Giordan, Kempa, Larkin, Linn, Lawson, Martinand, Matthews, McDermott, Novak, Nustraum, Ogborn, Osborne, Penick, Posner, Reif, Resnick, Schibeci, Solomon, Tiberghien, Tobin, Viennot, Wiltrok, Yager y tantos y tantos otros son nuestros contemporáneos. Y lo mismo podemos decir de la mayoría de los grandes encuentros, como el de La Londe de 1983, los congresos sobre preconcepciones organizados por Novak en Cornell, etc.

Más significátiva aún es la evohución de la visión de los propios investigadores: a principios de los 80 todavía se hablaba de la didáctica de las ciencias como de un dominio preparadigmático, preteórico. En cambio, como señala Hodson (1992): Hoy ya es posible construir un cuerpo de conocimientos en el que se integren coherentemente los distintos aspectos relativos a la enseñanzal aprendizaje de las ciencias.

Todo apunta pues, a un rápido desarrollo de una nueva comunidad científica, de un nuevo campo de investigación (Aliberas, Gutiérrez e Izquierdo, 1989).

\section{LA IMPORTANCIA DE LOS ORÍGENES}

Al señalar $\epsilon 1$ salto cualitativo que se produce en la década de los 80 no estamos minusvalorando el trabajo realizado en la etapa precedente, de desarrollo mucho más lento. Por el contrario, hemos de ser conscientes de que, si se desea reforzar el carácter de cuerpo específico de conocimientos (en construcción) de la didáctica de las ciencias y superar tratamientos ateóricos, es preciso tener en cuerita las distintas orientaciones, su capacidad para resistir a las modificaciones (lo que es un índice de una cierta coherencia y efectividad) y, en particular, 
resaltar su contribución al surgimiento de nuevos planteamientos.

Se trata de romper con una superficial adscripción a la última tendencia, como si de modas pasajeras se tratara (Gil 1986), o a un ecléctico «todo vale». Ello sigue constituyendo un peligro realque responde a una concepción de la didáctica como simple recopilación de recursos o de búsqueda de recetas.

Debemos así reconocer que, desde el punto de vistá de la investigación, cl movimiento de aprendizaje por descubrimiento no puede despacharse -como a menudo hemos hecho (Gil 1983, Hodson 1985, Millar y Driver 1987...)con una simple referencia a sus negativos resultados, a su fracaso en favorecer un aprendizaje de las ciencias más efectivo, a la denuncia de su inductivismo extremo, falta de atención a los contenidos, insistencia en una actividad completamente autónoma de los alumnos, etc. No podemos olvidar que este movimiento supuso un elemento dinamizador de una enseñanza que permanecía anciada en tradiciones asumidas acríticamente y constituyó el origen de reestructuraciones posteriores, dirigiendo la atencion hacta las concepciones subyaccntes acerca de la ciencia, provocando una revisión crítica de las mismas, etc. Mucho más importante que sus errores, insistimos, es el hecho de haber iniciado un proceso de innovación $e$ investigación sistemático en el que permanecemos inmersos (Gil 1993). Resulta esencial comprender esto porque muchos intentos de innovacion actuales reinciden habilalmente -y no por casualidad- en los mismos o similares errores a los del movimiento de aprendizaje por descubrimiento. Sin embargo, hay que saludar esos trabajos de innovación, al margen de su validez intrínseca, por lo que suponen de incorporación a un proceso de cuestionamiento y renovación que, una ve $z$ iniciado, facilitará el contacto con la literatura, es decir, con el trabajo de otros equipos y, en definitiva, el avance hacia las tesis consensuadas por la comunidad científica.

Lo mismo puede decirse de las propuestas de aprendizaje por recepción significativa (Ausubel 1978, Novak 1979): el hecho de contemplar la enseñanza-aprendizaje de las ciencias como un proceso de transmision-recepción no permite su descalificación como un simple retorno a Ia cstrategia «tradicional» (vista, a su vez, como algo sin ningún valor). No podemos olvidar algunas aportacio. nes clave como la iciea de aprendizaje significativo, la atención a aquello que los alumnos ya saben oinstrumentos como los mapas conceptuales y la Ve epistemológica de Gowin (Moreira y Novak 1988, Novak y Gowin 1989).

La atención a aquelio que los alumnos ya saben está, precisamente, en el origen de la «sorprendente sorpresa» que supuso el reconocimiento de que conceptos básicos y reiteradamente enseñados no llegaban a ser correctamente comprendidos y resultaban incapaces de desplazar las visiones intuitivas con las que los estudiantes Ilegaban at aula. Sorprendente, en primer lugar, por la importancia que iba a adquirir el estudio de las «preconcepciones» durante la década de los $80 \mathrm{y}$, en segundo lugar, porque, como había ya señalado muchos años antes Bachelard (1938), «resulta sorprendente que los profesores de ciencias no comprendan que los alumnos no comprendan». Los profesores deberíamos saber tener en cuenta -en opinión de Bachelard-que la vida cotidiana ha acumulado obstáculos contra los que se han de construir los conocimientos científicos. Pero, naturalmente, los profesores de ciencias no leíamos a Bachelard ni tenf́amos, en esa época, excesivas preocupaciones sobre las dificultades de aprendizaje o enseñanza de nuestra materia.

Llegamos así a los 80 , es decir, a la década que nos ocupa; una década durante la cual la línea de investigación prioritaria ha sido, precisamente, el estudio de las preconcepciones.

\section{EL ESTUDIO DE LAS PRECONCEPCIONES COMO LINEA DE INVESTIGACION PRIORITARIA}

No hay duda de que el estudio de lo que se conoce como "preconcepciones», «concepciones alternativas», «concepciones espontáneas»... ha constituido la línea prioritaria de investigación en didáctica de las ciencias durante la década de los 80 . Buen índice de ello es el porcentaje de artículos publicados, de comunicaciones presentadas en los congresos o de tesis doctorales sobre dicha temática. Ya hemos hecho referencia al estudio realizado por Duit (1993), que muestra el crecimiento auténticamente esponencial de este dominio, con más de 2.500 trabajos publicados durante esta década.

Laurence Viennot (1989), en una interesante revisión de la investigación francesa, ha intentado explicar el porqué de la abundancia de estudios en este campo. Además de la importancia dada a la descripción del uestado inicial» de los alumnos, Viennot se refiere a otra razón más pragmática: las investigaciones sobre ideas intuitivas, representaciones, etc. dan lugar a resultados más claros y convincentes que otros estudios; y, ante la necesidad de convencer en un periodo razonable de la efectividad de la investigación didáctica, muchos investigadorcs se han centrado en este campo.

Las cuestiones fundamentales, sin embargo, son: ¿qué ha aportado esta investigación sobre preconcepciones y qué perspectivas ha abierto? Vale la pena, sin duda, proceder a una reflexión detenida en torno a estas cuestiones si queremos sacar el máximo provecho de una investigación que ha ocupado mayoritariamente a la comunidad científica durante los uiltimos años. Intentaremos recoger, muy esquemáticamente, las principales aportaciones que, en nuestra opinión, son las siguientes:

- En primer lugar, la investigación sobre concepciones alternativas ha cuestionado con rotundidad la eficacia de la enseñanza por transmisión de conocimientos elaborados y, más generalmente, ha contribuido a cuestionar las visiones simplistas sobre el aprendizaje y la enseñanza de las ciencias, como la idea docente espontánea de que enseñar es una actividad simple para la cual basta con conocer la materia y algo de experiencia. Es preciso resaltar esta capacidad cuestionadora de la investigación sobre preconcepciones, puesto que ha contribuido más que 
cualquier otro estudio a problematizar la enseñanza/ aprendizaje de las ciencias, incluido el nivel universitario (Viennot 1989b), y a romper con la inercia de una tradición asumida acríticamente.

El número de trabajos publicados sobre preconcepciones en la práctica totalidad de los campos científicos es muy elevado, como muestra la amplia bibliografía publicada por Pfundt y Duit (1993), que recoge cerca de 3.000 trabajos, o las numerosas revisiones y selecciones bibliográficas publicadas en Enseñanza de las Ciencias (Carrascosa 1983 y 1985, Furió 1986, Cervantes 1987, Jiménez-Aleixandre 1987, Serrano 1987, Perales y Nievas 1988, Manrique, Varela y Favieres 1989, Camascosa y Gi1 1992, Grau 1993...).

Insistimos en la naturaleza crítica de estos estudios, que han mostrado la escasa efectividad de la enseñanza habitual en los distintos dominios cientfficos y han abierto perspectivas de renovación. Particular importancia han tenido, en ese sentido, las numerosas tesis doctorales que han abordado en profundidad campos concretos. Enseñanza de las Ciencias ha reseñado buen número de dichas tesis, que constituyen un instrumento imprescindible para proseguir la investigación en este campo.

- Esta investigación ha mostrado una gran capacidad integradora, conectando con los estudios sobre lenguaje (Llorens, De Jaime y Llopis 1989), epistemología genética (Pozo y Carretero 1982, Laburú y Pessoa de Carvalho 1992), etc.

- Ha favorecido, en particular, el encuentro con los planteamientos constructivistas, que se han convertido en el consenso emergente en la ensefianza de las ciencias (Resnick 1983, Driver 1986, Novak 1988) y han sido calificados como la aportación más relevante de las últimas décadas para la enseñanza/aprendizaje de las ciencias (Gruender y Tobin 1991).

- Ha dirigido la atención hacia las aportaciones de la historia y filosofía de las ciencias, como ya muestra, por ejemplo, el clásico trabajo de Posner, Strike, Hewson y Gertzog (1982); atención que ha experimentado un auténtico «boom» a finales de los 80 (Matthews 1990 y 1994, Izquierdo 1992) con la publicación de monográficos en las principales revistas internacionales.

- Ha provocado la génesis de nuevos modelos de enseñanza/aprendizaje de las ciencias: cambio conceptual (Posner et al. 1982, Nusbaum y Novick 1982, Driver y Oldham 1986, Pozo 1989)); aprendizaje generativo (Osborne y Wittrock 1983 y 1985); cambio conceptual, metodológico y actitudinal (Gil y Carrascosa 1985, Gil et al. 1991); cambio de comprensión - «shift understanding》-(Hodson 1988); aprendizaje alostérico (Giordan 1989)... Por lo demás, esta diversidad de denominacionestrecubre una convergencia básica que apunta a concebir el aprendizaje como el resultado de una investigación dirigida a partir del tratamiento de problemas que puedan interesar a los alumnos y alumnas (Gil et al. 1991, Gil 1993, Porlán 1993).
- Ya a finales de los 80, ha dirigido la atención hacia las preconcepciones docentes y epistemológicas del profesorado (Geni, y Gil 1987, Shuell 1987, Hewson y Hewson 1988). Se ha Ilegado a comprender, en efecto, que de la misma forma que los alumnos tienen preconcepciones que juegan un papel esencial en el proceso de aprendizaje y que es preciso tener en cuenta al planificar la enseñanza, los futuros profesores poseen ya una serie de concepcione;, hábitos y actitudes docentes «de sentido común», debidos a una larga impregnación ambiental durante el peciodo en que fueron alumnos. La influencia de esta formatión «incidental» es enorme porque responde a experiencias reiteradas y se adquiere de forma no reflexiva corto algo natural, obvio, «de sentido común», escapando a.sí a la crítica y convirtiéndose en un obstáculo.

Vemos, pues, que la investigacion sobre preconcepciones ha resultado muy fructífera y ha abierto perspectivas que siguen mereciendo atención. Pero habría que preguntarse también sobre los posibles inconvenientes de esta casi exclusiva atención a las preconcepciones de los alumnos y la consiguiente necesidad de reorientar la investigación.

\section{CONTRA EL REDUCCIONISMO CON. CEPTUAL.}

A finales de la década de los 80 -aunque algunas voces aisladas se habían manifestado antes (Gil y Carrascosa 1985, Hashweh 1986)-se comienza a comprender que la atención casi exclusiva hacia las preconcepciones con olvido de otros aspectos igualmente relevantes para el aprendizaje dle las ciencias supone un grave reduccionismo (Duschl y Gitomer 1991), que explicaría las limitaciones de las estrategias de cambio conceptual (Shuell 1987. White y Gunstone 1989).

Dushl y Gitomer han criticado una cierta visión jerárquica del cambio zonceptual, según la cual los cambios conceptuales producirán cambios simultáneos de procedimientos y actitudes. Se ha empezado así a tener en cuenta que la consirucción de conocimientos científicos tiene exigencias metodológicas y epistemológicas a las que es preciso prestar atención explícita. Ello ha proporcionado nuevo sentido e interés a las investigaciones sobre trabajos de laboratorio y resolución de problemas de lápiz y papel, vistos ahora como instrumentos de familiarización cle los alumnos con las estrategias del trabajo científico. Y esto, a su vez, exige una profunda transformación de dichas prácticas y problemas, para que dejen de ser meros ejercicios de aplicación o ilustraciones de los zonocimientos transmitidos verbalmente y pasen a constituir situaciones problemáticas abiertas, capaces de favorecer una actividad investigadora de los alumnos, convenientemente orientada y apoyada por el profesor (Gil et al. 1991, Wheatley 1991, Git et al. 1992, Hodson 19si2b).

Se ha empezado del mismo modo a comprender que la construcción de conocimientos científicos tiene también exigencias axiológicas y que, por decirlo de una forma gráfica, no se puede pensar en implicar a los alumnos y 
alumnas en una tarea investigadora... bajo un clima de control policial. Y al decir esto, no estamos exagerando demagógicamente, sino recogiendo expresiones utilizadas por los propios profesores, que se sienten incómodos en ese papel de control y de exigencia desconfiada que impone, aparentemente, la actividad docente (Briscoe 1991). Ello ha proporcionado nuevo sentido e interés a las investigaciones sobre:

- Relaciones CTS. La construcción de conocimientos está asociada al tratamiento de problemas de interés para Ios alumnos (Gil et al. 1991, Gil 1993, Porlan 1993), a la toma de decisiones (Aikenhead 1985) y, en definitiva, a la adopción de actitudes responsables y fundamentadas frente al desarrollo científico/técnico y las consecuencias que se derivan (Catalán y Catany 1986, Polo y López 1987, Solbes y Vilches 1989, Jiménez y Otero 1990 , Solomon 1990).

- Actitudes del alumnado (y del propio profesorado). Aparecen como un factor determinante del proceso de aprendizaje (Escudero 1985, Yager y Penick 1986) y cabe esperar que se vean favorecidas por tratamientos contextualizados, relevantes, por formas de trabajocreativas, por formas de evaluación que impulsen y apoyen y no se limiten a sancionar (Gil et al.1991).

- Clima del aula y del centro. La importancia de estos factores ambientales (Welch 1985, Rivas 1986) aparece con claridad desde la perspectiva constructivista, que contempla el aprendizaje de las ciencias como una actividad próxima a una investigación realizada por «investigadores novatos» bajo la dirección de un experto (Gil 1993).

Todos estos estudios están dejando de ser tratamientos desconexos para apoyarse mutuamente y contribuir a la construcción de un cuerpo coherente de conocimientos (Hodson 1992). Se ha comprendido, por ejemplo, que ninguna innovación puede consolidarse mientras la evaluación no se transforme en el mismo sentido (Linn 1987). No se puede pretender, en efecto, que los estudiantes construyar conocimientos mediante una actividad proxima a una investigación dirigida y seguir contemplando la evaluacion como un «juicio objetivo» y terminal de la tarea realizada por cada alumno. Por el contrario, como "director se investigadores noveles», el profesor ha de considerarse corresponsable de los resultados que éstos obtengan; to puede situarse frente a ellos, sino con ellos; su pregunia no puede ser «quién merece una valoración positiva y quién no» sino «qué ayudas precisa cada estudiante para seguir avanzando y alcanzar los logros deseados» (Gil et aI. 1991). Ello obliga a prestar a la evaluación del proceso de enseñanza/aprendizaje de las ciencias una atención que hasta aquí ha sido muy insuficiente (Geli 1986, Colombo, Pesa y Sainas 1986, Alonso, Gil y Martínez-Torregrosa 1992).

Comienza a vislumbrarse así la emergencia de un modelo de aprendizaje de las ciencias que abarca coherentemente todos los aspectos del proceso de enseñanza/aprendizaje y que apunta hacia la transformación de la actividad de Ios alumnos en una tarea investigativa. Como ha resumido Hodson (1992), la reciente investigación didáctica está mostrando que los estudiantes desarrollan mejor su comprensión conceptual y aprenden más acerca de la naturaleza de la ciencia cuando participan en investigaciones cientificas, provisto que haya suficientes oportunidades y apoyo para la reflexión.

Podría pensarse que esta idea de aproximar el aprendizaje de las ciencias a un trabajo científico no es en absoluto novedosa, sino que constituye una vieja intuición, $\tan$ vieja como el movimiento de «aprendizaje por descubrimiento», cuyos resultados, por lo demás, dejaron mucho que desear (Ausubel 1978). Sin embargo, Jas propuestas actuales difieren de aquéllas en dos aspectos fundamentales que conviene puntualizar:

En primer lugar es preciso subrayar que no se concibe a los alumnos como investigadores autónomos trabajando en la frontera del conocimiento. Esta metáfora-que fue propuesta como reacción a la que concebía a los alumnos como simples receptores- presenta graves limitaciones y no resuita útil para organizar el trabajo de los alumnos (Gil 1993). La metáfora actual que presenta a los estudiantes como investigadores noveles permite, en nuestra opinión, una mejor comprensión de la situación de aprendizaje, integrando coherentemente las aportaciones de Vygotski sobre el papel del adulto (profesor) en el proceso de enseñanza/aprendizaje, etc. (Gil 1993).

La segunda diferencia esencial entre las propuestas actuales de aprendizaje de las ciencias como investigación y las de aprendizaje por descubrimiento estriba en el intento de superación de las visiones deformadas acerca de la naturaleza del trabajo cientifico, muy extendidas entre el profesorado y con una notable incidencia sobre la práctica docente (Désautels et al. 1993). Dedicaremos el siguiente apartado a esta cuestión que aparece hoy como uno de los elementos clave para la renovación de la enseñanza de las ciencias.

\section{LAS CONCEPCIONES ESPONTÁNEAS SOBRE LA CIENCIA COMO OBSTÁCULO FUNDAMENTAL}

Existe un consenso creciente sobre que, si se quiere cambiar lo que los profesores y alumnos hacemos en las clases de ciencias, es preciso previamente modificar la epistemología "espontánea» de los profesores (Bell y Pearson 1992, Désautels et al. 1993). Esta epistemología espontánea aparece así como un obstáculo fundamental capaz de bloquear los intentos de renovación. Y no se trata tan sólo de criticar el inductivismo extremo tantas veces denunciado (Gil 1983, Hodson 1985, Millar y Driver $1987 \ldots$ ), es preciso prestar atencion a muchas otras deformaciones y reduccionismos como los que resumimos a continuación (Gil 1993):

- Visión empirista y ateórica: Se resalta el papel de la observación y de la experimentación «neutras» (no contaminadas por ideas apriorísticas»), olvidando el papel esencial de las hipotesis y de Ia construcción de un cuerpo coherente de conocimientos (teoría). 
Por otra parte, pese a esta importancia dada (verbalmente) a la observación y experimentación, en general la enseñanza es puramente libresca, sin apenas trabajo experimental. Ello favoreceque la experimentación conserve para profesores y alumnos el atractivo de una "revolu* ción pendiente».

Se incide particularmente en esta visión ateórica cuando se presenta el aprendizaje de la ciencia como una cuestión de descubrimiento o se reduce a la práctica de los procesos con olvido de los contenidos.

- Visión rígida (algorítmica, «exacta», infalible...). Se presenta el «método científico» como conjunto de etapas a seguir mecánicamente. Se resaita, por otra parte, lo que supone tratamiento cuantitativo, control riguroso, etc., olvidando -o incluso rechazando- todo lo que significa invención, creatividad, duda...

- Visión aproblemática y ahistórica (ergo dogmática). Se transmiten conocimientos ya elaborados, sin mostrar cuáles fueron los problemas que generaron su construcción, cuál ha sido su evolución, las dificuitades, etc., ni mucho menos aún las limitaciones del conocimiento actual o las perspectivas abiertas.

- Visión exclusivamente analítica. Resalta la necesaria parcialización de los estudios, su carácter acotado, simplificatorio, pero olvida los esfuerzos posteriores de unificación y de construcción de cuerpos coherentes de conocimientos cada vez más amplios, el tratamiento de problemas «frontera» entre distintos dominios que pueden llegar a unirse, etc.

- Visión acumulativa, lineal. Los conocimientos apare. cen como fruto de un crecimiento lineal, ignorando las crisis, las remodelaciones profundas. Se ignora, en particular, la discontinuidad radical entre el tratamiento científico de los problemas y el pensamiento ordinario.

- Visión "velada», elitista. Se esconde la significación de los conocimientos tras el aparato matemático. No se hace un esfuerzo por hacer la ciencia accesible, por mostrar su carácter de construcción humana, en la que no faltan ni confusión ni errores... como los de los propios alumnos.

En el mismo sentido, se presenta el trabajo científico como un dominio reservado a minorías especialmente dotadas, transmitiendo expectativas negativas hacia la mayoría de los alumnos, con claras discriminaciones de naturaleza social y sexual (la ciencia es presentada como una actividad eminentemente «masculina»).

- En el polo opuesto nos encontramos con una Visión de "sentido común». Los conocimientos se presentan como claros, obvios, "de sentido común», olvidando que la contrucción científica parte, precisamente, del cuestionamiento sistemático de lo obvio.

Se contribuye implícitamente a esta visión cuando se practica el reduccionismo conceptual, es decir, cuando se presenta el paso de las concepciones alternativas de los alumnos a los conocimientos científicos como sim. ple cambio de ideas, sin tener en cuenta los cambios metodológiccs que exige dicha transformación, o sea, cuando se ignoran las diferencias substanciales que existen entre el pensamiento de sentido común y el tratamiento científico de los problemas.

- Visión individualista. Los conocimientos científicos aparecen corro obra de genios aislados, ignorándose el papel del trabajo colectivo, de los intercambios entre equipos... Se teja creer, en particular, que los resultados de un sólo científico o equipo pueden verificar o falsar una hipótesis.

- Visión descontextualizada, socialmente neutra. Se olvidan las complejas relaciones CTS y se proporciona una imagen de los científicos como seres «por encima del bien y del mal», encerrados en torres de marfil y ajenos a las necesarias tomas de decisión.

Como reacción puede caerse en una visión excesiva. mente sociologista de la ciencia que diluye completamente su especificidad, así como en planteamientos muy simplistas: exaltación de la ciencia como factor absoluto de progreso o rechazo sistemático (a causa de su capacidad destructiva, efectos contaminantes, etc.).

Numerosos estudios están mostrando la extenșión de estas visiones deformadas entre los profesores y alumnos (Carrascosa, Fernández, Gil y Orozco 1993, Meichstry 1993) y la necesidad de iransformarlas. Debemos, sin embargo, salir al paso de una visión pesimista al respecto: 4 s cierto que los profesores tenemos concepciones, comportamientos y actitudes que constituyen auténticos obstáculos para una actividad docente efectiva. Pero es preciso también señalar que basta con proporcionar a los profesores la ocasión de un trabajo de reflexión y profundización para que sus puntos de vista se aproximen a los resultados de la investigación diđáctica.

En otras palabras: el peligro real estriba en la falta de atención a aquello que se adquiere incidental y acríticamente por impregnación ambiental, y no en una especial dificultad del profesorado para superar las deformaciones habituales. Esta superación aparece asociada a diversas líneas de investigación: desde el estudio del pensamiento docente espontáneo (Gil 1991, Bell y Pearson 1992, Porlan 1993) al análisis de la orientación de los trabajos prácticos (Hcdson 1992b), pasando por la consideración de las impitcaciones de la Historia y Filosofía de la ciencia en la enseñanza (Matthews 1990 y 1994). Se está contribuyendo así a una mejor comprensión del trabajo científico y a una más correcta orientación del aprendizaje como construcción de conocimientos científicos.

Podemos corcluir, pues, que la investigación en didáctica de las ciencias ha experimentado un desarrollo particularmente fructífero durante la pasada década, iniciándose la construcción de un cuerpo específico de conocimientos en torno a los problemas de enseñanza/ aprendizaje de las ciencias. Cabe ahora esperar que dicho cuerpo de conocimientos se desarrolle y consolide en los próximos años, haciendo posible un efectivo desplazamiento del modelo de transmisión/recepción por las orientaciones constructivistas, tanto en la activi- 
dad del atumnado como en la formación y actividad del profesorado. Ésa es Ia perspectiva generaI que detallaremos, para finalizar, en el siguiente apartado.

\section{PERSPECTIVAS DE DESARROLLO PARA UNA NUEVA DECADA}

Un análisis retrospectivo como el que acabamos de realizar tiene como objetivo fundamental favorecer una recapitulación integradora que proporcione perspectivas para futuros desarrollos. El enunciado de dichas perspectivas - a caballo entre la predicción más o menos "objetiva» y la recomendación claramente interesadaconstituye un elemento esencial de las revisiones como la que aquí hemos intentado y se convierte en hipótesis de trabajo que permitirá contrastar la validez del análisis realizado y las conclusiones extraídas.

Podemos considerar, a título de ejemplo, la revisión realizada por Welch (1985) hace aproximadamente una década. En un análisis muy amplio y riguroso del volumen. de investigación realizada en distintos campos de la didáctica de las ciencias y de los resultados alcanzados, Welch intentó contestar a las siguientes cuestiones, claramente prospectivas:

- ¿Qué es lo que conocemos en el presente y qué deberíamos conocer?

\section{- ¿Cuáles son las investigaciones más urgentes?}

- ¿Cómo podemos mejorar la enseñanza de las ciencias?

Nada garantiza, por supuesto, que el resultado de estos análisis sea correcto; pero incluso, si las conclusiones son cuestionables, su caracter explícito permite posicionarse frente a las mismas y enunciar «hipótesis alternativas", es decir, otras predicciones y recomendaciones. Así, al reseñar el trabajo de Welch (Enseñanza de las Ciencias, Vol, 4(1), pp. 65-67), manifestamos nuestro acuerdo con bastantes de sus conclusiones (como las que señalaban la importancia de las investigaciones, aún poco desarrolladas, sobre las actitudes de los alumnos y las influencias ambientales o sobre el tipo de actividades realizadas por los alumnos); pero expusimos también nuestras dudas acerca de otros resultados y recomendaciones: «Digamos por nuestra parte -escribfamos- que, pese a ser un trabajo dt: indudable interés, algunas de sus conclusiones nos parecen escasamente fundamentadas y contrastan con los resultados de las investigaciones más recientes. En particular, Welch no parece haber percibido la importancia de las investigaciones centradas en los preconceptos y esquemas conceptuales alternativos de los alumnos [... . También es cuestionable su insistencia en que lo que los profesores hacen no es relevante. ¿Acaso lo que los alumnos hacen no depende del comportamiento del profesorado, al impulsar un tipo u otro de actividades? ¿Acaso las actitudes de los alumnos hacia una ciencia no dependen muy a menudo de las de sus profesores y del tipo de aprendizaje que favorecen?...».
Hoy nos reafirmamos en estas reflexiones críticas. De hecho, todos los análisis indican que los estudios sobre preconcepciones -ignorados por Welch-se han convertido en una Ínea de investigación prioritaria, mientras que los estudios sobre el profesorado -con una nueva orientación basada en el cuestionamiento del pensamiento docente espontáneo-han comenzado a desarrollarse con fuerza. Pero insistimos en el interés que la recapitulación de Welch tuvo para nuestro trabajo, y ello no «a pesar de las discrepanciasnsino, muy particularmente, al permitir posicionarnos en contra de algunas de sus tesis. Deseariamos que las perspectivas que vamos a enunciar ahora -basándonos en el análisis realizado en los apartados precedentes- tuvieran también la virtualidad, cuanto menos, de favorecer predicciones y propuestas alternativas. He aquí, pues, nuestras predicciones sobre el desarrollo, en los próximos años, de la investigación en didáctica de las ciencias:

\begin{abstract}
- En primer lugar cabe esperar una acentuación de la búsqueda de coherencia global que conduzca al desarrollo y consolidación del cuerpo de conocimientos específico sobre problemas de enseñanza/aprendizaje de las ciencias iniciado esta pasada década hasta llegar a integrar de forma coherente desde los estudios sobre introducción y manejo de conceptos a los de evaluación.
\end{abstract}

Conviene precisar que, al poner el acento en la construcción de un cuerpo específico de conocimientos, no estamos preconizando planteamientos cerrados que ignoren las aportaciones de otros campos como el de fia psicología de la educación o el de la filosofía de la ciencia. Muy al contrario, es precisamente en la medida en que ese cuerpo de conocimientos existe, que resulta posible inspirarse en esas aportaciones sin caer en aplicaciones mecánicas y descontextualizadas, escasamente efectivas (Gil 1993b).

- En segundo lugar, y por lo que se refiere a la línea de investigación sobre preconcepciones, hoy prioritaria, creemos necesario un desplazamiento de las investigaciones sobre detección de preconcepciones (utilizando instrumentos que recogen, en general, aquello que los estudiantes responden de forma inmediata frente a determinadas cuestiones) hacia el estudio de las zonas de desarrollo potencial (recogiendo aquello que los alumnos pueden llegar a responder cuando se favorece su reflexión crítica). Dicho de otro modo, los resuitados acumulados sobre preconcepciones muestran, en nuestra opinión, una aproximación superficial a las situaciones (Carrascosa y Gil 1985) que los propios instrumentos de detección favorecen. Por ello nuestra hipotesis es que, tan importantes o más que dichos resultados, son aquéllos que muestran lo que los alumnos pueden llegar a hacer en condiciones favorables. Y pensamos que este tipo de estudio irá desplazando en los próximos años a los de simple detección de preconcepciones.

- Una evolución de las investigaciones sobre preconcepciones como la que acabamos de predecir/proponer es solidaria de otra transformación fundamental: la superación del reduccionismo conceptual que ha marcado hasta aquí, en buena medida, estas investigaciones, así como las propuestas de tratamiento. La toma en conside- 
ración simultánea de los aspectos conceptuales, procedimentales y actitudinales favorecerá la efectividad de las propuestas constructivistas y un consenso creciente en torno al modelo de aprendizaje como investigación.

- Asociado a este consenso en tomo a las orientaciones constructivistas, es previsible un fuerte incremento de los trabajos de innovación, dirigido a la elaboración de programas de actividades, es decir, programas de investigación dirigiđa, para orientar la construcción de conocimientos. De hecho este esfuerzo innovador tiene sólidas raíces en nuestro país y son numerosos los grupos que están contribuyendo ya a la elaboración de materiales didácticos en una perspectiva constructivista (Gil y Martínez-Torregrosa 1987, Gutiérrez y Rodríguez 1987 , Albadalejo, Caamaño et al. 1988, Hierrezuelo et al. 1988, Yus 1988, Calatayud et al. 1990, Gené 1991 ...). La orientación explícitamente constructivista del nuevo currículo recién aprobado en España (Coll 1989) ha impulsado notablemente estas tareas de innovación fundamentadas en los resultados de la investigación didáctica (Del Carmen 1990, Bernal et al. 1993, Caamaño et al. 1993, Cañal et al. 1993, Martín y Porlan 1993, Martínez-Torregrosa et al. 1993, Nieda y Barahona 1993, Rojero 1993, Varela et al. 1993...).

- Cabe, por otra parte, esperar una extensión de los planteamientos constructivistas a la formación del profesorado, con una creciente atención al pensamiento docente espontáneo, la orientación de dicha formación como (re)construcción de los conocimientos didácticos a través de una actividad investigadora, etc.

- Por último, aunque la universidad ha permanecido hasta aquí, en general, al margen de los procesos de renovación de la enseñanza de las ciencias, cabe esperar que a lo largo de la próxima década se produzca el despegue de la didáctica de las ciencias en el nivel universitario. Todo apunta, en efecto, a que estamos asistiendo a los comienzos de un movimiento semejante al que tuvo lugar en la enseñanza media hace dos décadas, debido a las mismas razones: rápido crecimiento de la

\section{REFERENCIAS BIBLIOGRÁFICAS}

ALIBERAS, J., GUTIÉRREZ, R. e LZQUIERDO, M. 1989. La didàctica de les ciencies: una empresa racional, Enseñanza de las Ciencias, 7(3), pp. 277-284.

ALBALADEJO, E. y CAAMAÑO, A. et al., 1988. La Química Faraday y La Física Faraday. (Teide: Barcelona).

ALONSO, M., GIL, D. y MARTÍNEZ-TORREGROSA, J., 1992. Concepciones espontáneas de los profesores de ciencias sobre la evaluación. Obstáculos a superar y propuestas de repianteamiento, Revista de Enseñanza de la Física, 5(2), pp. 18-38.

BACHELARD, G., 1938. La formation de l'esprit scientifique. (Vrin: París). población estudiantil (con lo que los estudios universitarios están dejando de ser la opción de pequeñas minorías), resultados muy preocupantes en lo que se refiere a índices de abandono o duración de los estudios, etc. Se está generando aśí una preocupación, todavía incipiente, en el profeso::ado universitario, por la renovación de la docencia, que esperamos experimente un impulso sensible a lo largo de los próximos años. De este modo, el proceso de renovación de la enseñanza de las ciencias y, muy concretamente, la formación inicial del profesora" do, dejaría de verse lastrado, durante los años decisivos de la formacićn universitaria, por una formación ambiental que se sitúa en las antípodas de las tendencias actuales.

No se nos escapa que nuestras predicciones resultan notablemente: optimismas y que algunos de los esperamos tienen una gran carga de deseamos. Pero si algo hemos aprendido acerca de la naturaleza del trabajo científico, es que ningún planteamiento es neutro y que las hipótesis e incluso los problemas que decidimos estudiar están influidos, adımás de por nuestros conocimientos, por nuestra ideología e intereses. Dicho de otro modo: sin deseos y expectativas no pueden producirse los avances que anunciamos, porque no se realizaría el trabajo necesario. Pero creemos que se trata de deseos y expectativas compartidos, en torno a los cuales está produciéndose un consenso creciente. Esperemos que, dentro de otra década, la investigación didáctica y la situación de la enseñanza de las ciencias confirmen, parcialmente al menos, estas expectativas.

\section{NOTA}

* Ponencia pesentada al IV Congreso Internacional sobre Investigación en la Didáctica de las Ciencias y de las Matemáticas, celebrado en Barcelona los días 13 al 16 de septiembre de 1993.
BELL, B.F. y F'EARSON, J, 1992. Better Learning, Intemational Journal of Science Education, 14(3), pp. 349-361.

BERNAL, M. GALLÁSTEGUł, J., JMÉNEZ, M.P. y OTERO, L., 1993. Aprendiendo a mirar hacia dentro: el proyecto ACES, Ensiñanzade las Ciencias, númeroextra(IV Congreso), pp. 29-30.

BRISCOE, C., 1991. The dynamic interactions among beliefs, role methaphores and teaching practices. A case study of teacher chinge, Science Education, 75(2), pp.185-199.

CAAMAÑO, A., et al., 1993. El projecte Gaia: una proposta per a l'ensenyament de les ciències 12-16, Enseñanza de las Ciencias, número extra (IV Congreso), pp. 33-34. 
CALATAYUD, M.L. y GIL, D. et al., 1990. La construcción de las ciencias físico-quimicas. Libro del alumno y libro del profesur. (Ed. Libreria NAU libres: València)

CAÑAL, P., LLEDÓ, A.I. y MERINO, I., 1993. Ciencias y conocimiento del medio: los ámbitos de investigación en el proyecio curricular «Investigando nuestro mundo» (Primaria), Enseñanza de las Ciencias, Número extra (IV Congreso), pp. $39-40$.

CARRASCOSA, J., 1983. Errores conceptuales en la enseñanza de las ciencias: Seleccion bibliográfica, Enseñanza de las Ciencias, 1, pp. 63-65.

CARRASCOSA, J., 1985. Errores conceptuales en la enseñanza de la física y la química: una revisión bibliográfica, Enseñanza de las Ciencias, 3(3), pp. 230-234.

CARRASCOSA. J., FERNÁNDEZ, I., GIL, D. y OROZCO, A., 1993. Análisis de algunas visiones deformadas sobre la naturalcza de la ciencia y las características del trabajo cientifíco, Enseñanza de las Ciencias, número extra (IV Congreso), pp. 43-44.

CARRASCOSA, J. y GIL, D., 1985. La metodologia de la superficialitat it'aprenentatge de les ciències, Enseñanza de las Ciencias, 3(2), pp. 113-120.

CARRASCOSA, J. y GIL, D., 1992. Concepciones alternativas en mecánica, Enseñanza de las Ciencias, 10(3), pp. 314-328.

CARRASCOSA. J., GIL, D. y MARTÍNEZ-TERRADES, F, 1993. Análisis cuantitativo del desarrollo de la investigación en didáctica de las ciencias. (Documento de trabajo).

CATALÁN, A. y CATANY, M., 1986. Contra el mito de la neutralidad de la ciencia: el papel de la historia, Enseñanza de las Ciencias, 4(2), pp. 163-166.

CERVANTES, A., 1987. Los conceptos de calor y temperatura: una revisión bibliográfica, Enseñanza de las Ciencias, 5, pp. $66-70$.

COL.L, C., 1989. Diseño Curricular Base y Proyectos Curriculares, Cuadernos de Pedagogia, 168, pp. 8-14.

COLOMBO, L., PESA, M. y SALINAS, J., 1986. La realimentación en la evaluación de un curso de laboratorio de física, Enseñanza de las Ciencias, 4(2), pp. 122-128.

DELCARMEN, L., 1990. La elaboración de proyectos curriculares de centro en el marco de un currículo de ciencias abierto, Enseñanza de las Ciencias, 8(1), pp. 37-45.

DÉSAUTELS, J., LAROCHELLE, M., GAGNÉ, B. y RUEL, F. 1993. La formation a l'ensetgnement des sciences: le virage épistémologiquıe, Didaskalia, 1, pp. 49-67.

DRIVER, R., 1986. Psicología cognoscitiva y esquemas conceptuales de los alumnos, Enseñanza de las Ciencias, 4(1), pp. 3-15.

DRIVER, R. y OLDHAM, V., 1986. A constructivist approach to curriculum development in science, Studies in Science Education, 13, pp. 105-122.

DUYT, R, 1993. Research on students' conceptions. Developments \& trends. Paper presented at the «Third International Seminar on Misconceptions and Educational Strategies in Science and Mathematics». Cornell University. Ithaca. EEUU.
DUSCHL, R. y GIIOMER, D., 1991. Epistemological perspectives on conceptual change: implications for educational practice, Journal of Research in Science Teaching, 28(9), pp. 839858.

ESCUDERO, T., 1985. Las actitudes en la enseñanza de las ciencias: un panorama complejo, Revista de Edtcación, 278. pp. 5-25

FURIÓ, C., 1986. Metodología utilizada en la deteccion de dificultades y esquemas conceptuales en la enseñanza de la química, Enseñanza de las Ciencias, 4(1), pp. 73-77.

FURIÓ, C., 1994. Tendencias actuales en la formación del profesorado de ciencias, Enseñanza de las Ciencias, 12(2), pp. 188-199.

GELI, A.M., 1986. L'avaluació de la biologia en la segona etapa d'EGB. Tesis doctoral. Universitat Autonoma de Barcelona.

GENÉ, A., 1991. Cambio conceptual y metodologico en la enseñanza y el aprendizaje de la evolución de los seres vivos. Un ejemplo concreto, Enseñanza de las Ciencias, 9(1), pp. $22-28$.

GENÉ, A. y GIL, D., 1988. La formación del profesorado como cambio didáctico, Revista Interuniversitaria de Formación del Profesorado, 2, pp. 155-159.

GIL, D., 1982. El profesorado y la investigación educativa. Primeras Jornadas de Investigación Didáctica en Fisica y Química, pp. 537-540. (ICE: València).

GIL. D., 1983. Tres paradigmas básicos en la enseñanza de las ciencias, Enseñanza de las Ciencias, 1(1), pp. 26-33.

GIL, D., 1986. El aprendizaje como investigación: ¿Nuevo modelo o nuevo eslogan superficial?, Actas de las IV Jornadas sobre la Investigación en la Escuela (Sevilla), pp. 330-334.

GIL, D., 1991. ¿Qué han de saber y saber hacer los profesores de ciencias?, Enseñanza de las Ciencias, 9(1), pp. 69-77.

GIL, D., 1993. Contribución de la historia y filosofía de las ciencias al desarrollo de un modelo de enseñanza/aprendizaje como investigación, Enseñanza de las Ciencias, 11(2), pp. $197-212$

GIL, D., 1993b. Psicología educativa y didáctica de las ciencias: los procesos de enseñanza/aprendizaje de las ciencias como lugar de encuentro, Infancia y Aprendizaje, 62-63.

GIL, D. y CARRASCOSA, J., 1985. Science learning as a conceptual and methodological change, European Journal of Science Education, 7(3), pp. 231-236.

GIL, D., CARRASCOSA, J., FURIÓ, c. y MARTÍNEZTORREGROSA, J., 1991. La enseñanza de las ciencias en la educación secundaria. (Horsori: Barcelona).

GIORDAN, A., 1989. De las concepciones de los alumnos a un modelo de aprendizaje alostérico, Investigación en la Escuela, 8, pp. 3-14.

GRAU, R., 1993. Revisión de concepciones en el área de la evolución, Enseñanza de las Ciencias, 11(1), pp. 87-89.

GRUENDER, C.D. y TOBIN, K., 1991. Promise and Prospect, Science Education, $75(1)$, pp. 1-8.

GRUP RECERCA, 1980-82. Proyecto Faraday. (ICEUniversitat Autònoma: Barcelona). 
GUTIÉRREZ, F.A. y RODRÍGUEZ, L. M., 1987. El aprendizaje te la física como investigacion: un ejemplo de aplicación en la enseñanza media, Enseñanzade las Ciencias, 5(2), pp. 135. 144.

HASHWEH, M.Z., 1986. Towards an explanation of conceptual change, European Journal of Science Education, 8(3), pp. 229-249.

HEWSON, P.W. y HEWSON, M.G., 1988. On appropriate conception of teaching science: a vjew from studies of science learning, Science Education, 72(5), pp. 597-614.

HIERREZUELO, J, et al., 1988. Aprendizaje en física y quimica: Programas guía de actividades para los alumnos y programas guía para el profesor. (Elzevir: Vélez Málaga. Málaga).

HODSON, D., 1985. Philosophy of science, science and science cducation, Studies in Science Education, 12, pp. 25-57.

HODSON, D., 1988. Towards a phylosophically more valid science curriculum, Science Education, 72(1), pp. 19-40.

HODSON, D., 1992. In search of a meaningful relationship: an exploration of some issues relating to integration in science and scicnce education, International Journal of Science Education, 14(5), pp. 541-566.

HODSON, D., 1992b. Assessment of practical work: some considerations in Philosophy of Science, Science \& Education, 1(2), pp. 115-144.

IZQUIERDO, M., 1992. Reconsidering the science curriculum starting from contemporary (converging) models of science and cognition: a research program. Proceedings of the Second International Conference on Hystory and Philosophy of Science in Science Teaching, pp. 517-529. (Kingston, Canadá).

IIMENEZ, M.P., 1987. Preconceptos y esquemas conceptuales en biología, Enseñanza de las Ciencias, 5(2), pp. 165-167.

JIMÉNEZ, M.P. y OTERO, L., 1990. Laciencia comoconstrucción social, Cuadernos de Pedagogía, 180, pp. 20-22.

LABURÚ, C.E. y PESSOA de CARVAL.HO, A., 1992. Investigación del desarrollo y aprendizaje de la noción de aceleración en adolescentes, Enseñanza de las Ciencias, 10(1), pp. 63-72.

LINN, M.C., 1987. Establishing a research base for science education: challenges, trends and recommendations, Journal of Research in Science Teaching, 24(3), pp. 191-216.

LLORENS, J.A., DE JAIME, M.C. y LLOPIS, R., 1989. La función del lenguaje en un enfoque constructivista del aprendizaje de las ciencias, Estseñanza de las Ciencias, $7(2)$, pp. 111-119.

MANRIQUE, M.J., VARELA, P. y FAVIERES, A., 1989. Selección bibliográfica sobre esquemas alternativos de los estudiantes en electricidad, Enseñanza de las Ciencias, 7(3), pp. 292-295.

MAR'I'́N, J. y PORLÁN, R., 1993. Los ámbitos de investigación escolar: una propuesta didáctica para la organización del conocimiento escolar en el marco del proyecto curricular IRES, Ensefianzade las Ciencias, númeroextra (IV Congreso), pp. 93-94.

MARTÍNEZ-TORREGROSA, J., et al., 1993. La búsqueda de la unidad (La estructura de todas las cosas'). Libro del profesor y libro del alumno. (Aguaclara: Alicante).
MATTHEWS, M.R., 1990. History, Philosophy and Science Teaching: A Rapprochement, Studies in Science Education, 18 , pp. $25-51$.

MATTHEWS, M.R., 1994. Historia, Filosofía y Enseñanza de las Ciencias: la aproximación actual, Enseñanza de las Ciencias, 12(2), pp. 255-277.

MCDERMOTT, L.C., 1984. Research on conceptual understanding in mechanics, Physics Today, Julio, pp. 24-34.

MEICHSTRY, Y., 1993. The impact of science curricula on students vietvs about the nature of science, Joumal of Research in Science Teaching, 39(5), pp. 429-443.

MILLAR, R. y DRIVER, R., 1987. Beyond processes, Studies in Science Education, 14, pp. 33-62.

MOREIRA, M.A., 1994. Diez años de la revista Enseñanza de las Ciencia:, Enseñanza de las Ciencias, 12(2), pp. 147-153.

MOREIRA, N.A. y NOVAK, D.P. 1988. Investigación en enseñanza de las ciencias en la universidad de Cornell: esquemas téricos, cuestiones centrales y abordes metodologizos, Enseñanza de las Ciencias, 6(I), pp. 3-18.

NIEDA, J. y BARAHONA, S., 1993. Ciencias de la Tierra y del Medio Ambiente. (Materiales didácticos). (MEC: Madrid).

NOVAK, J.D., 1979. The reception learning paradigm, Journal of Research in Science Teaching, 16, pp. 481-488.

NOVAK, J.D., 1988. Constructivismo humano: un consenso emergente, Entseñanza de las Cientias, 6(3), pp. 213-223.

NOVAK, J.D. y GOWIN, B., 1988. Aprendiendo a aprender. (M.Roca: Earcelona).

NUSBAUM, J. y NOVIK, S., 1982. Aiternative frameworks, conceptual sonflict and accommodation: towards a principled teaching strategy, Instructional Science, 11, P. 183-200.

OSBORNE, R. y WITTROCK, M., 1983. Learning Science: a generative process, Science Education, 67, pp. 490-508.

OSBORNE, R. y WITTROK, M., 1985. The generative learning model and ts implications for science education, Studies' in Science Education, 12 , pp. $59-87$

PERALES. F.I. y NIEVAS, F., 1988. Nociones de los alumnos sobre conceptos de óptica geométrica, Enseñanza de las Ciencias, $\mathrm{t}(1)$, pp. 86-89.

PFUNDT, H. y DUIT, R., 1993. Bibliography: Students'altemative frameworks and science education. (INP at the University of Kiel: Kiel, Alemania).

POLO, F. y LÓPEZ, J.A., 1987. Los científicos y sus actitudes políticas ante los problemas de nuestro tiempo, Enseñanza de las Ciercias, 5(2), pp. 149-156.

PORLÁN, R., 1993. Constructivismo y Escuela. Hacia un modelo de enseñanza-aprendizaje basado en la investigación. (Díada: Sevilla).

POSNER, G.I. et al., 1982. Accommodation of a scientific conception: towards a theory of conceptual change, Science Education, 66, pp. 211-227.

POZO, J.I., 1989. Teorías cognitivas del aprendizaje. (Morata: Madrid). 
POZO, I. y CARRETERO, M., 1982. Del pensamiento formaI a las concepciones espontáneas. ¿Qué cambia en la enseñanza de las ciencias?, Infancia y Aprendizaje, 38, pp. 35-52.

RESNICK, L.B., 1983. Mathematics and Science Learning: a new conception, Science, 220, pp. 477-478.

RIVAS, M., 1986. Factores de eficacia esolar: una línea de investigación didáctica, Bordón, 264, pp. 693-708.

ROJERO, F.F., 1993. Materiales didácticos para la educación ambiental en las ciencias de la naturaleza. Diseño y resultados, Enseñanza de las Ciencias, número extra (IV Congreso), pp. 185-186.

SERRAN(), T., 1987. Representaciones de los alumnos en biología: estado de la cuestion y problemas para su investigación en el aula, Enseñanza de las Ciencias, 5(3), pp. 181-188.

SHUELL, T.J., 1987. Cognitive psychology and conceptual change: implications for teaching science, Science Education, $7 \mathrm{I}(2)$, pp. 239-250.

SOLBES, J. y VILCHES, A., 1989. Interacciones CTS: un instrumento de cambio actitudinal, Enseñanza de las Ciencias, $7(1)$, pp. 14-20.

SOLOMON, I., 1990. The discussion of social issues in the science classroom, Studies in Science Education, 18, pp. $105-126$.
VARELA,P.,FAVIERES, A., MANRIQUE, M.J.V. y PÉREZLANDAZÁBAL, M.C., I993. Iniciación a la Física en el marco de la teoría constructivista, Enseñanza de las Ciencias, número extra (IV Congreso), pp. 289-290.

VIENNOT, L., 1989a. L'enseignement des sciences phisiques object de recherche, Bulletin de l'Union des Physiciens, 716 , pp. $899-910$.

VIENNOT, L., 1989b. La didáctica en la enseñanza superior, ¿para que? Enseñanza de las Ciencias, 7(1), pp. 3-13.

WELCH, W., 1985. Research in science education: review and recomendations, Science Education, 69, pp. 421-448.

WHEATLEY, G.H., 1991. Constructivist perspectives on Science and Mathematics learning, Science Education, 75(1), pp. 9-21.

WHITE, T.R. y GUNSTONE. F.R., 1989. Metalearning and conceptual change, International Journal Science Education, 11 , pp. $577-586$

YAGER, R.E. y PENICK, J.E., 1986. Perception of four groups towards science classes, teachers and value of science, Science Education, 70(4), pp. 335-363. 